hep-th/0310262

NSF-KITP-03-89

\title{
Exceptional Collections and del Pezzo Gauge Theories
}

\author{
Christopher P. Herzog \\ Kavli Institute for Theoretical Physics, \\ University of California, Santa Barbara, CA 93106, USA \\ herzog@kitp.ucsb.edu
}

\begin{abstract}
Stacks of D3-branes placed at the tip of a cone over a del Pezzo surface provide a way of geometrically engineering a small but rich class of gauge/gravity dualities. We develop tools for understanding the resulting quiver gauge theories using exceptional collections. We prove two important results for a general quiver gauge theory: 1) we show the ordering of the nodes can be determined up to cyclic permutation and 2) we derive a simple formula for the ranks of the gauge groups (at the conformal point) in terms of the numbers of bifundamentals. We also provide a detailed analysis of four node quivers, examining when precisely mutations of the exceptional collection are related to Seiberg duality.
\end{abstract}

October 2003 


\section{Introduction}

A convenient way of engineering $\mathcal{N}=1$ gauge/gravity dualities starts with a stack of D3branes placed at the tip of a Calabi-Yau cone $\mathbf{X}$ [1, 2, 3, 4, This construction generalizes the original AdS/CFT correspondence [5, 6, 7], where the D3-branes are placed in flat space, $\mathbf{X}=\mathbb{C}^{3}$. The resulting collection of gauge/gravity dualities is extremely rich; the number of qualifying $\mathbf{X}$ is infinite. Unfortunately, we lack a detailed understanding of most of these $\mathbf{X}$. For example, to the author's knowledge, the metric for only two such $\mathbf{X}$ is known, $\mathbb{C}^{3}$ and the conifold. In this paper, we will study dualities where $\mathbf{X}$ is a (complex) cone over a del Pezzo surface.

A del Pezzo surface is a two complex dimensional, Kaehler manifold with positive curvature. Two simple examples of del Pezzos are $\mathbb{P}^{2}$ and $\mathbb{P}^{1} \times \mathbb{P}^{1}$, for which we do know metrics. The corresponding $\mathbf{X}$ are orbifolds of $\mathbb{C}^{3}$ and the conifold. The remaining del Pezzos, denoted $d P_{n}$, correspond to $\mathbb{P}^{2}$ blown up at $n$ points where $1 \leq n \leq 8$.

Even though metrics for the $d P_{n}$ are lacking, these surfaces are extremely well studied and we can make progress in understanding their gauge/gravity dualities. For example, for $n=1,2,3$, the resulting $d P_{n}$ are toric and the gauge theories for these models can be extracted using toric geometry [8, 9, 10, 11].

Exceptional collections appear to be one of the most promising tools for understanding del Pezzo gauge theories. Exceptional collections exist for all del Pezzos. Given an exceptional collection, it is easy to generate another such collection through a braiding operation called mutation. For every exceptional collection, there is a prescription for writing down the quiver, the ranks of the gauge groups, and the R-charges of the fields at the conformal point. A brief review of these collections is provided in section 2 .

Exceptional collections were first proposed in this context by [12] although the authors provided detailed analysis only for toric cases. Later Wijnholt [13] developed this proposal, deriving gauge theories for $n>3 .^{1}$ Most recently, the author and Walcher [16] used exceptional collections to understand the dibaryon spectra in del Pezzo gauge theories, extending earlier work in 17,18 .

Nevertheless, our understanding of the connection between these collections and the del

\footnotetext{
${ }^{1}$ Other techniques have been proposed for deriving the gauge theory for $n>3$ : "unhiggsing" was suggested by [14] and $p q$-seven brane webs by [15].
} 
Pezzo gauge theories is far from complete. For example, it does not appear to be true that every exceptional collection generates a reasonable gauge theory. Certain collections generate gauge theories with gauge groups of zero rank and bifundamentals with negative R-charge! In this paper, we try to fill some of the gaps in our understanding.

One open problem is, given a quiver gauge theory for a del Pezzo, can we reverse engineer the corresponding exceptional collection? The exceptional collection is an ordered collection of sheaves, and each sheaf is identified with a node in the quiver. Thus to generate the exceptional collection, we have to order the nodes of the quiver.

In section 3, we partially solve this ordering problem. An exceptional collection, through a particularly simple set of mutations, can generate a bi-infinite sequence of sheaves called a helix. We prove that every quiver corresponds to a helix and that a cyclic permutation of the nodes corresponds to choosing a different foundation for the helix. This result reduces the full ordering problem to ordering the quiver up to cyclic permutation.

In section 4, we present a formula for the gauge group ranks that partially solves another part of the reverse engineering problem. In [16], these ranks were identified with the ranks of the bundles in the "dual" exceptional collection. Our formula depends only on the numbers of bifundamentals and an ordering of the quiver and thus can be used in constructing the "dual" collection.

In this paper, we rechristen the "dual" collection the geometric collection. The original exceptional collection we call the gauge theory collection.

Finally, in section 5, we present a detailed analysis of four node quivers for del Pezzos. We will see that there is only one such quiver, and only two orderings (up to cyclic permutation) are allowed. We denote these orderings $A$ and $F$ type. Seiberg duality of an $A$ type quiver produces another $A$ type quiver. Seiberg duality of an $F$ type quiver either produces a new $F$ type quiver or a quiver that cannot be described by an exceptional collection on a del Pezzo. Inspired by these results, we define a well split quiver to be an ordered quiver (corresponding to a helix) where Seiberg duality on any node is equivalent to a sequence of mutations. Our $A$ type quiver is well split. More generally, an unproven conjecture is that the Seiberg dual of a well split quiver is again well split. In contrast, the $F$ type quiver is $i l l$ split or equivalently not well split. Leaving precise definitions for the text, we believe it to be true that Seiberg duality cannot be expressed in terms of mutations for ill split quivers. 
Ultimately, we hope that the current work can be used to understand generalizations of the Klebanov-Strassler (KS) solution [19] where in addition to D3-branes, we add D5-branes wrapped on vanishing two-cycles in $\mathbf{X}$. These D5-branes take the theory away from the conformal point, and the gauge couplings begin to run. A qualitative understanding of this RG flow can be gained by performing a Seiberg duality every time a coupling diverges. For example, one can learn something about the change in the number of degrees of freedom as a function of RG scale. In this context, the analysis will be simpler if we restrict to well split quivers. We hope to return to these generalized KS flows in a future publication [20].

\section{Quivers from Exceptional Collections}

We review the construction of an $\mathcal{N}=1$ quiver gauge theory from an exceptional collection, as described in [13, 16. We will be interested in the class of gauge theories that are dual, via the AdS/CFT correspondence, to type IIB string theory in an $A d S_{5} \times \mathbf{Y}$ background, where $\mathbf{Y}$ is a $\mathrm{U}(1)$ bundle over a del Pezzo surface.

The starting point is an exceptional collection of sheaves $\mathcal{E}$ on a del Pezzo $d P_{m}, m=$ $0, \ldots, 8$. A standard mathematical reference for these collections is [21] (see also [22]). For the string theorist [12, 23], these sheaves are simply a set of elementary "rigid" branes generating all BPS configurations of the theory by bound state formation. There exist special maps between the sheaves denoted $\mathrm{Ext}^{i}$, which the string theorist may think of as the ground states of the strings connecting the elementary branes. For each sheaf, we have a $S U(N)$ gauge group where $N$ corresponds to how many of that particular type of brane we decided to include in the geometry. Moreover, for each $\mathrm{Ext}^{i}$ map, we have bifundamental matter fields.

\subsection{A Review of Exceptional Collections}

Having given the rough picture, we now become precise. Let $\mathbf{V}$ be a complex Fano variety, e.g. a del Pezzo. A sheaf $E$ over $\mathbf{V}$ is called exceptional if $\operatorname{Ext}^{0}(E, E)=\operatorname{Hom}(E, E)=\mathbb{C}$ and $\operatorname{Ext}^{k}(E, E)=0$ for $k>0$. An ordered collection of sheaves $\mathcal{E}=\left(E_{1}, E_{2}, \ldots, E_{n}\right)$ is called exceptional if each $E_{i}$ is exceptional and if, moreover, for each pair $E_{i}, E_{j}$ with $i>j$, we have $\operatorname{Ext}^{k}\left(E_{i}, E_{j}\right)=0$ for all $k$ and $\operatorname{Ext}^{k}\left(E_{j}, E_{i}\right)=0$ except possibly for a single $k$. 
To count the number of bifundamental fields in the gauge theory, we must understand these Ext maps. A useful tool is the generalized Euler character

$$
\chi(E, F)=\sum_{i}(-1)^{i} \operatorname{dimExt}^{i}(E, F)
$$

which by the Hirzebruch-Riemann-Roch theorem can be rewritten as

$$
\chi(E, F)=\int_{\mathbf{V}} \operatorname{ch}\left(E^{*}\right) \operatorname{ch}(F) \operatorname{Td}(\mathbf{V})
$$

where $\operatorname{ch}(E)$ is the Chern character of the sheaf $E$. Note that $\chi$ is a bilinear form. An additional useful fact for del Pezzos is that only $\operatorname{Ext}^{1}$ and $\operatorname{Ext}^{0}$ can be nontrivial [24]. This fact together with the Euler character allows one to compute the numbers of bifundamentals in the gauge theory quickly and easily from the Chern characters of the exceptional sheaves.

An obvious question at this point is how many sheaves do we include in the exceptional collection, or equivalently how many fundamental branes do we need to describe the physics. Geometrically, in these del Pezzos, branes can correspond to points, they can wrap curves, or they can wrap the entire del Pezzo. Thus, the number $n$ of sheaves in the collection should correspond to the sum of the Betti numbers of $d P_{m}, n=m+3$. Mathematically, we see that the Chern character $\operatorname{ch}(E)=\left(r(E), c_{1}(E), \operatorname{ch}_{2}(E)\right)$ is described by $n$ charges. At the level of Chern characters, we can have at most $n$ linearly independent sheaves in our collection. A complete exceptional collection contains $n$ sheaves and spans this $n$-dimensional vector space.

In components, the Euler character reads

$$
\begin{aligned}
\chi(E, F)= & r(E) r(F)+\frac{1}{2}(r(E) \operatorname{deg}(F)-r(F) \operatorname{deg}(E)) \\
& +r(E) \operatorname{ch}_{2}(F)+r(F) \operatorname{ch}_{2}(E)-c_{1}(E) \cdot c_{1}(F),
\end{aligned}
$$

which can easily be derived from (2) using $\operatorname{Td}\left(d P_{n}\right)=1-\frac{K}{2}+H^{2}$, where $K$ is the canonical class and $H$ is the hyperplane, with $\int_{d P_{n}} H^{2}=1$. Also the degree $\operatorname{deg}(E)=(-K) \cdot c_{1}(E)$.

If $\mathcal{E}$ is an exceptional collection, one obtains new exceptional collections (and hence new gauge theories) by left and right mutations:

$$
\begin{aligned}
L_{i}:\left(\ldots, E_{i-1}, E_{i}, E_{i+1}, \ldots\right) & \rightarrow\left(\ldots, E_{i-1}, L_{E_{i}} E_{i+1}, E_{i}, \ldots\right) \\
R_{i}:\left(\ldots, E_{i-1}, E_{i}, E_{i+1}, \ldots\right) & \rightarrow\left(\ldots, E_{i-1}, E_{i+1}, R_{E_{i+1}} E_{i}, \ldots\right)
\end{aligned}
$$


Here, $L_{E_{i}} E_{i+1}$ and $R_{E_{i+1}} E_{i}$ are defined by short exact sequences, whose precise form depends on which of the $\operatorname{Ext}^{k}\left(E_{i}, E_{i+1}\right)$ are non-zero. At the level of the Chern character

$$
\begin{aligned}
\operatorname{ch}\left(L_{E} F\right) & = \pm(\operatorname{ch}(F)-\chi(E, F) \operatorname{ch}(E)) \\
\operatorname{ch}\left(R_{F} E\right) & = \pm(\operatorname{ch}(E)-\chi(E, F) \operatorname{ch}(F))
\end{aligned}
$$

where the sign is chosen such that the rank of the mutated bundle is positive. We introduce some additional nomenclature here that will be important later on. If $\chi(E, F)<0$, the mutation is called an extension and the plus sign above is chosen. In the remaining cases, choosing the plus sign corresponds to a recoil while the negative sign is a division.

There are an additional class of mutations denoted $L^{D}$ and $R^{D}$, which at the level of charges leads to the selection of the plus sign in (5) above. Choosing the plus sign will lead often to sheaves with negative rank, which roughly speaking one may think of as the antibrane. For more details on mutations, see for example [21].

\subsection{Constructing the Gauge Theory}

We review the construction of a $\mathcal{N}=1$ superconformal gauge theory dual to string theory on $A d S_{5} \times \mathbf{Y}$ for $\mathbf{Y}$ a $\mathrm{U}(1)$ bundle over $d P_{m}$.

To construct the gauge theory, we begin with an exceptional collection $\mathcal{E}^{G}=\left(E_{1}^{G}, E_{2}^{G}, \ldots, E_{n}^{G}\right)$ over $d P_{m}$. The quiver will consist of $n$ nodes, one node for each $S U\left(N_{i}\right)$ gauge group. The ranks of the gauge groups are defined to be $N_{i}=r\left(E_{i}^{G}\right) N$. Such a collection $\mathcal{E}^{G}$ we will refer to as a geometric collection.

Next we construct the dual exceptional collection $\left(\mathcal{E}^{Q}\right)^{\vee}=\mathcal{E}^{G}$. For any exceptional collection $\mathcal{E}$, we define the dual collection $\mathcal{E}^{\vee}$ to be the result of a braiding operation,

$$
\begin{aligned}
\mathcal{E}^{\vee} & =\left(E_{n}^{\vee}, E_{n-1}^{\vee}, \ldots, E_{1}\right) \\
& =\left(L_{E_{1}}^{D} \cdots L_{E_{n-1}}^{D} E_{n}, L_{E_{1}}^{D} \cdots L_{E_{n-2}}^{D} E_{n-1}, \ldots, L_{E_{1}}^{D} E_{2}, E_{1}\right)
\end{aligned}
$$

The collection $\mathcal{E}^{\vee}$ is exceptional in the order presented, and is dual to $\mathcal{E}$ in the sense of the Euler form, i.e. $\chi\left(E_{i}, E_{j}^{\vee}\right)=\delta_{i j}$. Note that because of the D-type mutations involved, the ranks of $\mathcal{E}^{\vee}$ may not be all positive. We call this dual collection $\mathcal{E}^{Q}$ the gauge theory collection. Note that the superscript $\vee$ is meant only to indicate the dual. 
Third, we construct the incidence matrix

$$
S_{i j}=\chi\left(E_{j}^{Q}, E_{i}^{Q}\right) .
$$

From the exceptional property, $S$ will be an upper triangular matrix with integer entries and ones along the diagonal. The incidence matrix for the original geometric collection is the inverse of $S$. Note that $S^{-1}$ is also upper triangular, integer valued, and has ones along the diagonal.

We use $S$ to compute the numbers of bifundamentals. We draw $S_{i j}$ arrows from node $i$ to node $j$ in the quiver. (A negative $S_{i j}$ means we have to reverse the direction of the arrows.) Each arrow is a bifundamental $\mathcal{N}=1$ chiral superfield transforming under the gauge groups at the tail and head of the arrow. It is straightforward to verify that the chiral anomalies in the resulting gauge theory vanish:

$$
\sum_{j}\left(S-S^{T}\right)_{i j} r\left(E_{j}^{G}\right)=0 .
$$

Finally, we may compute the R-charges of the bifundamental fields. Let $i$ correspond to the tail of the arrow and $j$ to the head. Building on work of Intriligator and Wecht [18], Herzog and Walcher [16] showed that

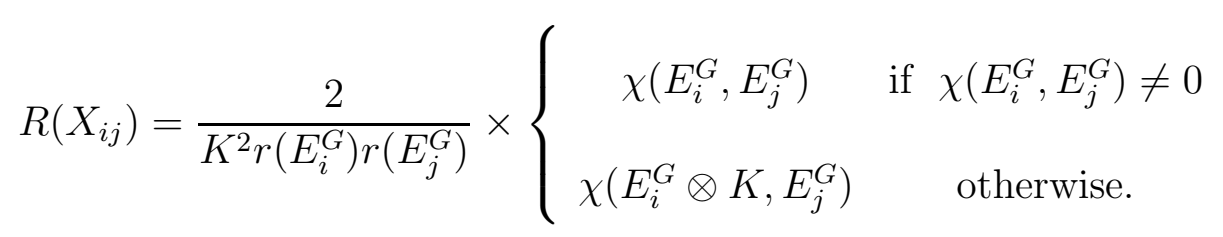

From these R-charges, one may verify that the NSVZ beta functions vanish. In particular, it was shown in [16 that

$$
0=\beta_{i}=\frac{1}{K^{2}} \sum_{k}\left(\chi\left(E_{i}^{Q}, E_{k}^{Q}\right)-\chi\left(E_{k}^{Q}, E_{i}^{Q}\right)\right)\left(\chi\left(E_{i}^{G}, E_{k}^{G}\right)-\chi\left(E_{k}^{G}, E_{i}^{G}\right)\right)+r\left(E_{i}^{Q}\right) r\left(E_{i}^{G}\right) .
$$

We can move away from the conformal point by adding fractional branes to the geometry. The fractional branes change the ranks of the gauge groups in a way that continues to preserve the cancellation of the chiral anomalies (8). Thus, these fractional branes correspond to the remaining vectors in the kernel of $\left(S-S^{T}\right)$.

\section{$3 \quad$ Helices and Quivers}

Having constructed a conformal gauge theory starting from an exceptional collection, it is natural to wonder whether an exceptional collection can be constructed from a conformal 
gauge theory. Figuring out the ordering of the nodes is clearly important. The quiver does not suggest any obvious ordering.

As a first step to solving the ordering problem, we will show that the choice of ordering should be independent of cyclic permutations of all the nodes. For example, for a four node quiver, the ordering (1234) should be equivalent to the ordering (2341). The proof requires introducing the notion of a helix.

A helix $\mathcal{H}=\left(E_{i}\right)_{i \in \mathbb{Z}}$ is a bi-infinite extension of an exceptional collection $\mathcal{E}$ defined recursively by

$$
\begin{aligned}
E_{i+n} & =R_{E_{i+n-1}} \cdots R_{E_{i+1}} E_{i} \\
E_{-i} & =L_{E_{i-1}} \cdots L_{E_{n-1-i}} E_{n-i} \quad i \geq 0
\end{aligned}
$$

such that the helix has period $n$, by which we mean

$$
E_{i}=E_{n+i} \otimes K \quad \forall i \in \mathbb{Z}
$$

A complete exceptional collection on a del Pezzo will generate a helix. Moreover, the helix remains the same (for our purposes) if constructed instead with $D$-type mutations. Any subcollection of $\mathcal{H}$ of the form $\left(E_{i+1}, E_{i+2}, \ldots, E_{i+n}\right)$ is exceptional and is called a foundation of $\mathcal{H}$.

Given a geometric collection $\mathcal{E}^{G}$ and the associated helix $\mathcal{H}$, a natural question is how does the gauge theory depend on the choice of foundation. The answer is that it doesn't. The gauge theory depends only on the choice of helix. In other words, for every helix, there exists a unique quiver. Moreover, shifting the foundation corresponds on the gauge theory side to a cyclic permutation of the nodes of the quiver.

Let $\mathcal{E}=\left(E_{1}, E_{2}, \ldots, E_{n}\right)$ and $\mathcal{F}=\left(E_{n} \otimes K, E_{1}, E_{2}, \ldots, E_{n-1}\right)$ be two neighboring geometric foundations of $\mathcal{H}$. Tensoring with $K$ does not affect the rank of the sheaf. Thus, the ranks of the gauge groups will be cyclically permuted but otherwise unchanged. We can prove that the quiver is independent of the helix by showing that the quivers constructed from the gauge theory collections $\mathcal{E}^{\vee}$ and $\mathcal{F}^{\vee}$ are identical.

Consider the dual exceptional collections $\mathcal{E}^{\vee}=\left(E_{n}^{\vee}, E_{n-1}^{\vee}, \ldots, E_{1}^{\vee}\right)$ and $\mathcal{F}^{\vee}$ :

$$
\mathcal{E}^{\vee}=\left(E_{n} \otimes K, L_{E_{1}}^{D} L_{E_{2}}^{D} \cdots L_{E_{n-2}}^{D} E_{n-1}, \ldots, L_{E_{1}}^{D} E_{2}, E_{1}\right)
$$


and

$$
\mathcal{F}^{\vee}=\left(E_{n-1} \otimes K, L_{E_{n} \otimes K}^{D} L_{E_{1}}^{D} L_{E_{2}}^{D} \cdots L_{E_{n-3}}^{D} E_{n-2}, \ldots, L_{E_{n} \otimes K}^{D} E_{1}, E_{n} \otimes K\right)
$$

To analyze these dual collections, we need a couple of lemmas. Let $G, E$, and $F$ be three exceptional sheaves. It follows from linearity of the Euler character $\chi$ and the definition of left mutation that

$$
\chi\left(L_{G}^{D} E, F\right)=\chi(E-\chi(G, E) G, F)=\chi(E, F)-\chi(G, E) \chi(G, F) .
$$

In the case $G=F$ and if $\chi(E, G)=0$, it follows that

$$
\chi\left(L_{G}^{D} E, G\right)=-\chi(G, E) .
$$

If $\chi(E, G)=0, \chi(F, G)=0$, and $\chi(F, E)=0$, it follows that

$$
\chi\left(L_{G}^{D} E, L_{G}^{D} F\right)=\chi(E, F) .
$$

Let $S_{i j}=\chi\left(E_{n+1-i}^{\vee}, E_{n+1-j}^{\vee}\right)$ and $T_{i j}=\chi\left(F_{n+1-i}^{\vee}, F_{n+1-j}^{\vee}\right)$ be $n \times n$ matrices constructed from $\mathcal{E}^{\vee}$ and $\mathcal{F}^{\vee}$ respectively. Consider the submatrix $\chi\left(E_{n+1-i}^{\vee}, E_{n+1-j}^{\vee}\right)=s_{i j}$ where $E_{i}^{\vee}$ and $E_{j}^{\vee}$ can be any sheaves in $\mathcal{E}^{\vee}$ except for $E_{n} \otimes K$. Let $t_{i j}$ be the corresponding submatrix for $\mathcal{F}^{\vee}$ where again we are not allowed to use $E_{n} \otimes K$. The submatrices $s$ and $t$ are identical as $(n-1) \times(n-1)$ dimensional matrices. This statement follows from (17).

Now consider the remaining entries in the $T$ and $S$ matrices. In particular, consider $S_{1 j}$ and $T_{(j-1) n}$ where $j=2,3, \ldots, n . \quad\left(S_{11}=T_{n n}=1\right.$ and the other entries vanish trivially because of the ordering inside the collection.) It follows from (16) that

$$
\begin{aligned}
T_{(j-1) n} & =\chi\left(L_{E_{n} \otimes K}^{D} L_{E_{1}}^{D} \cdots L_{E_{n-j}}^{D} E_{n-j+1}, E_{n} \otimes K\right) \\
& =-\chi\left(E_{n} \otimes K, L_{E_{1}}^{D} \cdots L_{E_{n-j}}^{D} E_{n-j+1}\right) \\
& =-S_{1 j} .
\end{aligned}
$$

Using the matrices $T$ and $S$ we can construct quivers. These quivers will be identical up to a cyclic permutation of the nodes. Clearly the quivers from the submatrices $s$ and $t$ must be identical. The minus sign in (18) then compensates for the cyclic permutation.

\subsection{Ordering and the Superpotential}

These del Pezzo gauge theories can have a superpotential which up to this point we have ignored. The superpotential, if known, further constrains the ordering of the nodes. 
The superpotential $W$ is a gauge invariant polynomial in the bifundamentals $X_{i j}$ of the quiver gauge theory. The superpotential generates relations in the path algebra of the quiver:

$$
\frac{\partial W}{\partial X_{i j}}=0
$$

Because of the R-symmetry, $W$ will have R-charge two. A typical term in the superpotential corresponds to a loop in the quiver. One multiplies the associated $X_{i j}$ together and traces over the color indices.

From the R-charge formula (9), it should be clear that a loop in the quiver will produce a monomial in the $X_{i j}$ with an R-charge that is a positive integer multiple of two [16]. Assume for the moment that we know the correct ordering of the quiver. If we take the convention where $i$ corresponds to the tail of the arrow and $j$ to the head, we get an additional two in the R-charge every time $j>i$ in the monomial. For example, $X_{43} X_{32} X_{21} X_{14}$ would have R-charge two while $X_{34} X_{42} X_{21} X_{13}$ would have R-charge four.

Working backward, we see that the order the nodes appear in monomials in the superpotential must be the same order in which the nodes appear in the exceptional collection. The superpotential is often enough to specify the order of the nodes in the collection up to cyclic permutation.

\section{The Ranks of the Gauge Groups}

Having partially solved the ordering portion of the inverse problem, we now derive a formula for the ranks of the gauge groups (or equivalently the ranks of the sheaves in the helix) from the numbers of bifundamentals. This formula can be used to constrain further the ordering as we will see in section 5 .

We assume the existence of a gauge theory collection $\mathcal{E}^{Q}=\left(E_{1}, \ldots, E_{n}\right)$. From the formula for the Euler character (3), we know that

$$
\chi\left(E_{1}^{\vee} \otimes K, E_{j}^{\vee}\right)=K^{2} r\left(E_{1}^{\vee}\right) r\left(E_{j}^{\vee}\right)-\chi\left(E_{j}^{\vee}, E_{1}^{\vee}\right) .
$$

Note that the Euler character is invariant under tensoring both sheaves with an invertible 
sheaf. Some standard manipulation gives

$$
\begin{aligned}
\chi\left(E_{1}^{\vee} \otimes K, E_{j}^{\vee}\right) & =\chi\left(E_{1}^{\vee}, E_{j}^{\vee} \otimes(-K)\right) \\
& =\chi\left(E_{1},\left(L_{E_{1}}^{D} \cdots L_{E_{j-1}}^{D} E_{j}\right) \otimes(-K)\right) \\
& =\chi\left(E_{1}, R_{E_{n}}^{D} \cdots R_{E_{j+1}}^{D} E_{j}\right) .
\end{aligned}
$$

Similarly for the other term in (20), one finds

$$
\begin{aligned}
\chi\left(E_{j}^{\vee}, E_{1}^{\vee}\right) & =\chi\left(L_{E_{1}}^{D} \cdots L_{E_{j-1}}^{D} E_{j}, E_{1}\right) \\
& =\chi\left(L_{E_{2}}^{D} \cdots L_{E_{j-1}}^{D} E_{j}, E_{1}\right)-\chi\left(E_{1}, E_{1}\right) \chi\left(E_{1}, L_{E_{2}}^{D} \cdots L_{E_{j-1}}^{D} E_{j}\right) \\
& =-\chi\left(E_{1}, L_{E_{2}}^{D} \cdots L_{E_{j-1}}^{D} E_{j}\right) .
\end{aligned}
$$

Putting these two small results together, one finds for (20) that

$$
K^{2} r\left(E_{1}^{\vee}\right) r\left(E_{j}^{\vee}\right)=\chi\left(E_{1}, R_{E_{n}}^{D} \cdots R_{E_{j+1}}^{D} E_{j}\right)-\chi\left(E_{1}, L_{E_{2}}^{D} \cdots L_{E_{j-1}}^{D} E_{j}\right)
$$

This formula (21) has a simple graphical interpretation. We put $n$ points on a circle and label them clockwise from one to $n$. The second term in (21) is a sum over paths from point one to point $j$, while the first term is a sum over paths from point $j$ to point one.

More precisely, let $\mathcal{P}_{a b}$ be the set of paths from $a$ to $b$, with $a<b$. Let $I \in \mathcal{P}_{a b}$ be a particular path. $I$ is a map from the set of integers $0 \leq j \leq m+1$ to the set of integers $a \leq k \leq b, I(j)=k_{j}$, such that if $i<j$ then $k_{i}<k_{j}$. Moreover, $k_{0}=a$ and $k_{m+1}=b$. Finally, the path length of $I$ is defined to be $C(I)=m$.

With this notation, we may write

$$
K^{2} r\left(E_{i}^{\vee}\right) r\left(E_{j}^{\vee}\right)=\sum_{I \in \mathcal{P}_{i, j} \cup \mathcal{P}_{j, i+n}}(-1)^{C(I)+1} \prod_{s=0}^{C(I)} x_{I(s), I(s+1)}
$$

where we have defined $x_{k l}=\left(S-S^{T}\right)_{k l}$ with $S_{k l}=\chi\left(E_{k}, E_{l}\right)$. We put the whole set of paths on a circle by identifying $x_{l, k+n}=-x_{k l}$. We have used the cyclic permutativity proved in the previous section to substitute an arbitrary node $i$ for 1 in (22). Indeed, the formula remains valid even when $i=j$, as we will see below.

To gain confidence that we have the right formula, we check that the chiral anomalies cancel

$$
K^{2} r_{i} \sum_{j=1}^{n} r_{j} x_{i j}=0
$$


where $r_{i}=r\left(E_{i}^{\vee}\right)$ are the ranks of the gauge groups. The $x_{i j}$ closes all the paths from $i$ to $j$ to make loops. We will see that this sum over loops vanishes in a trivial way. By a loop we mean an element $I \in \mathcal{P}_{j, j+n}$ together with the obvious equivalence relation. In particular, two paths $I \in \mathcal{P}_{j, j+n}$ and $I^{\prime} \in \mathcal{P}_{j^{\prime}, j^{\prime}+n}$ construct the same loop if they have the same length $C(I)=C\left(I^{\prime}\right)$ and when viewed on the circle, visit the same nodes in the same cyclic order.

Let $\mathcal{L}_{i}$ be the set of loops which include the node $i$. This set of loops can be decomposed into a sum over paths. In particular

$$
\mathcal{L}_{i}=\bigoplus_{k=1}^{n-1} \overline{\mathcal{P}_{i, i+k}}
$$

The overline notation means that we have closed the path into a loop by joining nodes $i$ and $i+k$. More precisely, let $I \in \mathcal{P}_{a b}$. Then $\bar{I} \in \mathcal{P}_{a, a+n}$. For $0 \leq j \leq m+1, I(j)=\bar{I}(j)$ and $\bar{I}(m+2)=a+n$. We can also perform this decomposition of loops into paths in a different way:

$$
\mathcal{L}_{i}=\bigoplus_{k=1}^{n-1} \overline{\mathcal{P}_{k+i, i+n}}
$$

Now we substitute (22) into (23) and switch the order of summation. We find that we have summed over all loops involving the node $i$ twice. The $x_{i j}$ in (23) introduces a relative minus sign between the two sums. Each loop in one sum pairs up with a loop in the other and cancels.

As a final check, we derive a formula for $K^{2} r_{1}^{2}$ using the NSVZ beta function $\beta_{1}$. As reviewed above, it was demonstrated in [16] that

$$
0=\beta_{1}=r\left(E_{1}^{\vee}\right)^{2}+\frac{1}{K^{2}} \sum_{k}\left(\chi\left(E_{1}, E_{k}\right)-\chi\left(E_{k}, E_{1}\right)\right)\left(\chi\left(E_{1}^{\vee}, E_{k}^{\vee}-\chi\left(E_{k}^{\vee}, E_{1}^{\vee}\right)\right)\right.
$$

where we have used the fact that $E_{1}^{\vee}=E_{1}$. This proof is more rigorous than it may seem. We are not assuming that the beta function vanishes in order to derive $r_{1}$. Rather, in [16], the right hand side of (26) was shown to vanish as a mathematical identity.

From the beta function then

$$
\begin{aligned}
K^{2} r_{1}^{2} & =\sum_{k} \chi\left(E_{1}, E_{k}\right) \chi\left(E_{k}^{\vee}, E_{1}^{\vee}\right) \\
& =-\sum_{k} \chi\left(E_{1}, E_{k}\right) \chi\left(E_{1}, L_{E_{2}}^{D} \cdots L_{E_{k-1}}^{D} E_{k}\right) .
\end{aligned}
$$


Using the path language, the sum is over all loops involving the node 1. Using cyclic invariance, we conclude that $K^{2} r_{i}^{2}$ is in general a sum over all loops involving the node $i$ :

$$
K^{2} r_{i}^{2}=\sum_{L \in \mathcal{L}_{i}}(-1)^{C(L)+1} \prod_{s=0}^{C(L)} x_{L(s), L(s+1)}
$$

Note that loops with even numbers of $x_{k j}$ appear with a plus sign. This formula is equivalent to (22) in the case $i=j$.

There is a simpler way of writing this sum. Formally, we may change the basis of the $S$ matrix.

$$
S \rightarrow B S B^{T}
$$

Although $S$ is not invariant under such a change of basis, $\operatorname{Tr} S^{-1} S^{T}$ is, and we can use this trace invariant. First we evaluate this trace invariant in a particularly simple basis. In particular, we choose the basis in which the sheaves are written in terms of their rank, first chern class, and second chern character, as in (3). It is straightforward to see that $\operatorname{Tr} S S^{-T}=n$. Evaluating $\operatorname{Tr} S S^{-T}$ in the exceptional collection basis, we find a sum over all loops in the quiver. In particular, the first row of $S$ times the first column of $S^{-T}$ is a sum over all loops involving node one plus a one from the diagonal elements. Then the second row of $S$ times the second column of $S^{-T}$ will be a sum over all loops involving node two but not involving node one again plus a one from the diagonal elements, and so on. Comparing $\operatorname{Tr} S S^{-T}$ in the two bases, we find that the sum over all loops must vanish,

$$
0=\sum_{L \in \mathcal{L}}(-1)^{C(L)} \prod_{s=0}^{C(L)} x_{L(s), L(s+1)} .
$$

Thus $K^{2} r_{i}^{2}$ may also be thought of as a sum over all loops not involving node $i$. (Now loops with even numbers of $x_{k j}$ will appear with a minus sign.)

As a coda to this section, we rewrite our formula for $K^{2} r_{i}^{2}$ in yet one more way. In particular, we produce the minor $S^{i}$ of $S$ by crossing out the $i$ th row and $i$ th column. For a quiver with $n+1$ nodes $(n<12)$,

$$
K^{2} r_{i}^{2}=-\operatorname{Tr}\left(S^{i}\right)^{-1}\left(S^{i}\right)^{T}+n
$$




\section{The Four Node Quiver}

We closely investigate the inverse problem for four node quivers. The starting point is a four node quiver with arrows joining all the nodes. In order to satisfy anomaly cancellation, at each node there must be either two arrows in and one arrow out or two arrows out and one arrow in (assuming no arrows vanish).

Our first lemma is that there is only one such quiver up to permutation of the nodes. We will draw this quiver as in figure 1. A brute force proof (which the writer employed) is to draw all the possibilities and relate them by permutation.

We will assume that this quiver corresponds to a geometric collection $\mathcal{E}^{G}=\left(E_{4}^{\vee}, E_{3}^{\vee}, E_{2}^{\vee}, E_{1}^{\vee}\right)$ with the ranks of the $E_{i}^{\vee}$ all positive and the numbers of bifundamentals determined from the dual gauge theory collection $\mathcal{E}^{Q}$. The nodes in the quiver, in some yet to be determined order, correspond to the sheaves and the arrows to the Ext groups between the sheaves in $\mathcal{E}^{Q}$.

A little bit of notation is in order. We have the matrix $S_{i j}=\chi\left(E_{i}, E_{j}\right)$. We write $S$ as

$$
S=\left(\begin{array}{llll}
1 & a & b & c \\
0 & 1 & d & e \\
0 & 0 & 1 & f \\
0 & 0 & 0 & 1
\end{array}\right)
$$

From the previous section, it is clear that we need only care about the order up to cyclic permutations. If the dual of $\mathcal{E}^{G}=\left(E_{4}^{\vee}, E_{3}^{\vee}, E_{2}^{\vee}, E_{1}^{\vee}\right)$ generates our quiver, then so will the dual of $\mathcal{F}^{G}=\left(E_{1}^{\vee} \otimes K, E_{4}^{\vee}, E_{3}^{\vee}, E_{2}^{\vee}\right)$.

Without loss of generality, we may choose the node in the upper left hand corner of figure 1 to be node number one. There are then six possible orderings of the remaining nodes, corresponding to the six possible permutations of three objects. We label these six permutations $A$ through $F$. We will use a small Roman numeral to denote the particular cyclic ordering. For example $A$ type labeling corresponds to a clockwise labeling of the nodes. Then $A i$ corresponds to the quiver with node one in the upper left and the nodes labeled clockwise, Aii with node one in the upper right, Aiii in the lower right, and Aiv in 

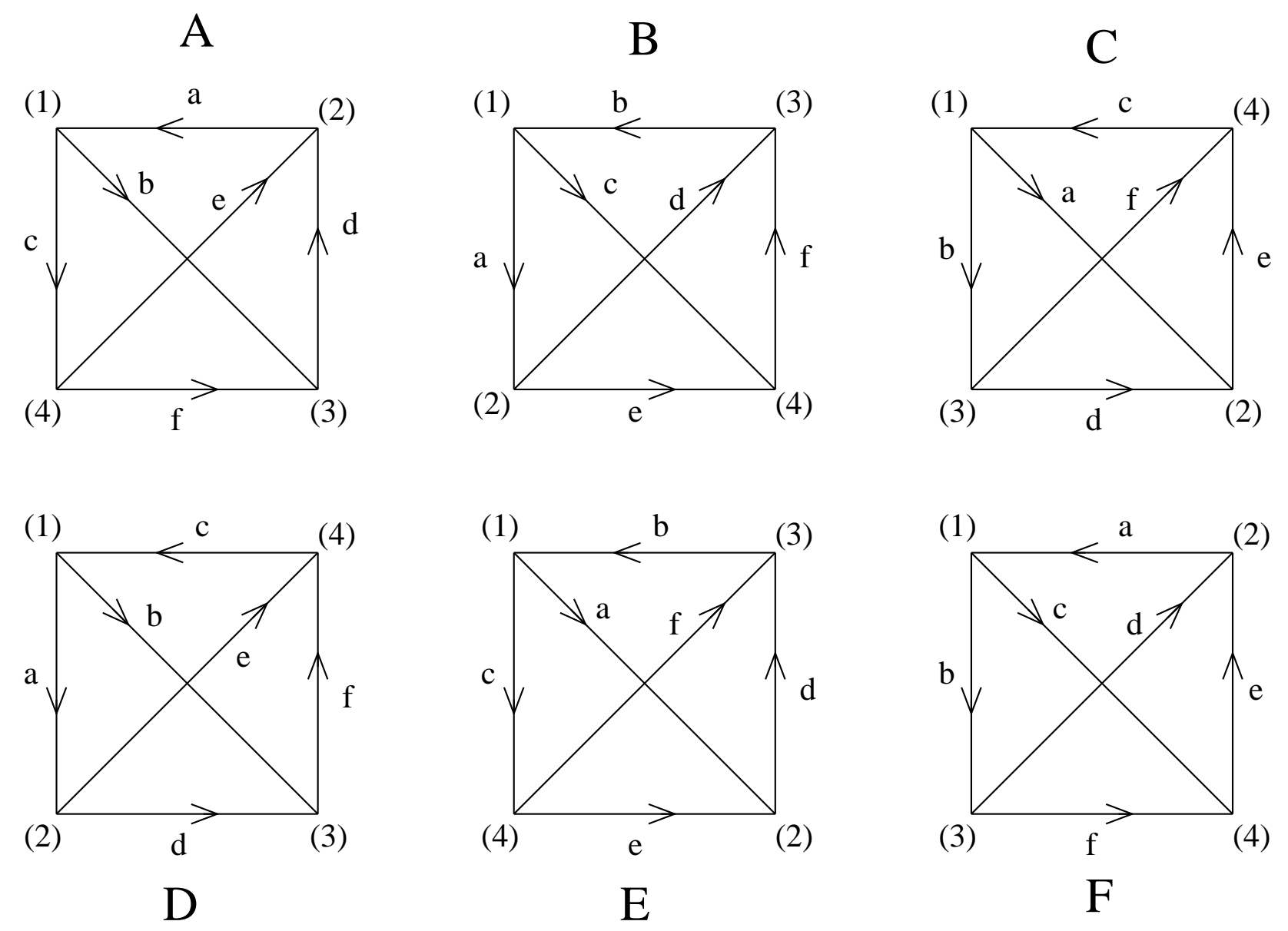

Figure 1: Different possible labelings of the four node quiver. 
the lower left. The $i$ type labelings always correspond to quivers with node one in the upper left and are shown in figure 1

These orderings give us information about the signs of the entries of the $S$ matrix. We can ask if these sign assignments are consistent with what we expect for an exceptional collection. We will find that only the $A$ and $F$ type labelings are allowed.

The sign assignments for the labelings are as follows

\begin{tabular}{|l|llll|}
\hline$A$ & $i$ & $i i$ & $i i i$ & $i v$ \\
\hline$a$ & - & - & - & - \\
$b$ & + & + & - & - \\
$c$ & + & + & + & + \\
$d$ & - & - & - & - \\
$e$ & - & + & + & - \\
$f$ & - & - & - & - \\
\hline
\end{tabular}

\begin{tabular}{|l|llll|}
\hline$B$ & $i$ & $i i$ & $i i i$ & $i v$ \\
$a$ & + & - & - & + \\
$b$ & - & - & + & + \\
$c$ & + & + & - & - \\
$d$ & + & + & - & - \\
$e$ & + & - & - & + \\
$f$ & - & + & + & - \\
\hline
\end{tabular}

\begin{tabular}{|l|llll|}
\hline$C$ & $i$ & $i i$ & $i i i$ & $i v$ \\
\hline$a$ & + & + & + & - \\
$b$ & + & - & - & + \\
$c$ & - & - & + & - \\
$d$ & - & + & + & + \\
$e$ & + & + & - & - \\
$f$ & + & - & + & + \\
\hline
\end{tabular}

\begin{tabular}{|l|llll|}
\hline$D$ & $i$ & $i i$ & $i i i$ & $i v$ \\
\hline$a$ & + & + & + & + \\
$b$ & + & - & - & + \\
$c$ & - & - & - & - \\
$d$ & + & + & + & + \\
$e$ & + & + & - & - \\
$f$ & + & + & + & + \\
\hline
\end{tabular}

\begin{tabular}{|l|cccc|}
\hline$E$ & $i$ & $i i$ & $i i i$ & $i v$ \\
\hline$a$ & + & - & - & + \\
$b$ & - & + & + & - \\
$c$ & + & + & - & - \\
$d$ & + & + & - & - \\
$e$ & - & - & + & + \\
$f$ & - & + & + & - \\
\hline
\end{tabular}

\begin{tabular}{|l|llll|}
\hline$F$ & $i$ & $i i$ & $i i i$ & $i v$ \\
\hline$a$ & - & - & + & - \\
$b$ & + & + & - & - \\
$c$ & + & - & + & + \\
$d$ & - & - & - & + \\
$e$ & - & + & + & - \\
$f$ & + & - & - & - \\
\hline
\end{tabular}

We begin by eliminating the $C$ type labeling. From the arguments presented above, we know that $\operatorname{Tr} S^{-1} S^{T}=4$ is an invariant of the exceptional collection. Evaluating $\operatorname{Tr} S^{-1} S^{T}$ for arbitrary $S$, we find that

$$
a^{2}+b^{2}+c^{2}+d^{2}+e^{2}+f^{2}-a b d-a c e-b c f-d e f+a c d f=0 .
$$


From the sign assignments in (32), one can see that every term in this trace invariant (33) will be positive for the $C$ labeling and that this trace invariant can never vanish. Thus, we eliminate the $C$ labeling.

A little more information can be gleaned from our change of basis. In the chern character basis, $S-S^{T}$ is clearly of rank two. The rank will not change under change of basis. Thus we conclude that

$$
c d-b e+a f=0
$$

Every sign assignment in (32) is consistent with (34) so we will have to go farther afield to eliminate labelings $B, D$, and $E$.

Our next step is to establish the ranks of the gauge groups, or equivalently the ranks of the bundles in $\mathcal{E}^{G}$. We can use the general formulae presented above. For example, (30) tells us that

$$
\begin{aligned}
& 8 r_{1}^{2}=d^{2}+e^{2}+f^{2}-d e f \\
& 8 r_{2}^{2}=b^{2}+c^{2}+f^{2}-b c f \\
& 8 r_{3}^{2}=a^{2}+c^{2}+e^{2}-a c e \\
& 8 r_{4}^{2}=a^{2}+b^{2}+d^{2}-a b d
\end{aligned}
$$

In establishing the ordering, we found the bilinears of the form $r_{i} r_{j}, i \neq j$ more useful. The equation (22) gives

$$
\begin{aligned}
& 8 r_{1} r_{2}=c d f-b d-c e, \\
& 8 r_{1} r_{3}=a d-c f, \\
& 8 r_{1} r_{4}=a e+b f-a d f, \\
& 8 r_{2} r_{3}=a c f-a b-e f, \\
& 8 r_{2} r_{4}=-a c+f d, \\
& 8 r_{3} r_{4}=a c d-d e-b c .
\end{aligned}
$$

The rank formulae (36) allow us to rule out orderings $B, D$, and $E$. Because the ranks of the gauge groups (and of the sheaves in $\mathcal{E}^{G}$ ) must be positive, the right hand side of the relations (36) must be positive. The sign assignments of $B, D$, and $E$ would force some ranks to be negative. 
We have succeeded in reducing the 24 different orderings of the nodes to two possible orderings, the $A$ and $F$ type orderings. Typically, to distinguish between the two orderings, it is enough to figure out which ordering satisfies (33).

We can figure out the allowed terms in the superpotential by looking at the R-charges of the bifundamentals. A term in the superpotential corresponds to a loop in the quiver where we trace over the internal indices of the bifundamentals. Looking at the four node quiver,

there are two triangular loops and one square loop that could produce scalar superpotential terms. The superpotential terms must have R-charge two. As discussed in section 3.1, the R-charge of a loop will be twice the number of times neighboring nodes are not in descending order as we go around the loop. For the $A$ type quiver, all three terms are allowed. However, for the $F$ type quiver, the square loop will have R-charge four and cannot appear in the superpotential.

\subsection{The Cubic R-charge Anomaly}

With these ranks, one can check the value of the cubic R-charge anomaly for the four node quivers. For $\mathcal{N}=1$ superconformal gauge theories, the conformal anomaly [25, 26] is

$$
a_{c}=\frac{3}{32}\left(3 \operatorname{Tr} R^{3}-\operatorname{Tr} R\right) \text {. }
$$

The vanishing of the NSVZ beta functions tells us that $\operatorname{Tr} R=0$. From AdS/CFT, we expect 6. 27, 28,

$$
a_{c}=\frac{\operatorname{Vol}\left(\mathbf{S}^{5}\right)}{4 \operatorname{Vol}(\mathbf{Y})}
$$

where $\mathbf{Y}$ is either a $U(1)$ bundle over $\mathbb{P}^{1} \times \mathbb{P}^{1}$ or $d P_{1}$. In both cases, we know that $\operatorname{Vol}(\mathbf{Y})=$ $8 \pi^{3} / 27$ [29], and so we expect that $\operatorname{Tr} R^{3}=3$.

The R-charges we know from (9):

$$
\begin{aligned}
\operatorname{Tr} R^{3}= & \sum_{i=1}^{4} r_{i}^{2}+a\left(1-R_{a}\right)^{3} r_{1} r_{2}+b\left(1-R_{b}\right)^{3} r_{1} r_{3}+c\left(1-R_{c}\right)^{3} r_{1} r_{4} \\
& +d\left(1-R_{d}\right)^{3} r_{2} r_{3}+e\left(1-R_{e}\right)^{3} r_{2} r_{4}+f\left(1-R_{f}\right)^{3} r_{3} r_{4}
\end{aligned}
$$

where

$$
R_{a}=\frac{-2 a}{r_{1} r_{2}}, \quad R_{d}=\frac{-2 d}{r_{2} r_{3}}, \quad R_{f}=\frac{-2 f}{r_{3} r_{4}}
$$




$$
\begin{gathered}
R_{b}=\frac{2(a d-b)}{r_{1} r_{3}}, \quad R_{e}=\frac{2(d f-e)}{r_{2} r_{4}}, \\
R_{c}=\frac{2(-c+a e+b f-a d f)}{r_{1} r_{4}} .
\end{gathered}
$$

This expression for Tr $R^{3}$ is valid for both $A$ and $F$ type quivers. The expression is rather cumbersome, even for computer aided algebra, but with some care, we were able to verify that $\operatorname{Tr} R^{3}=3$ when subject to the constraints (33) and (34).

Note that these "R-charges" $R_{a}, R_{b}$, and so on are not the true R-charges. Depending on the sign of $a, b$, etc., the $R_{a}, R_{b}$, etc. are either the R-charge or two minus the R-charge. For example, for the $A i$ quiver $R\left(X_{21}\right)=R_{a}, R\left(X_{32}\right)=R_{d}, R\left(X_{43}\right)=R_{f}$, and $R\left(X_{42}\right)=R_{e}$. However, $R\left(X_{13}\right)=2-R_{b}$ and $R\left(X_{14}\right)=2-R_{c}$.

We expect the R-charges to be positive, and it is interesting to investigate whether the constraints so far considered enforce this positivity. Indeed from the sign assignments (32) it is clear that for the $A i$ quiver, $R\left(X_{21}\right), R\left(X_{32}\right)$, and $R\left(X_{43}\right)$ are all positive. It is straightforward, using (32) and the rank formulae (36) to show that the remaining R-charges are also positive.

For the $F$ type quivers, the R-charges may in general be negative, as we will now see. Take the Fii quiver: $R\left(X_{21}\right)=R_{a}, R\left(X_{32}\right)=R_{d}, R\left(X_{43}\right)=R_{f}$, and $R\left(X_{41}\right)=R_{c}$ while $R\left(X_{13}\right)=2-R_{b}$ and $R\left(X_{24}\right)=2-R_{e}$. While it is straightforward to show that $R\left(X_{21}\right)$, $R\left(X_{32}\right), R\left(X_{43}\right)$, and $R\left(X_{41}\right)$ are positive, the remaining two $\mathrm{R}$-charges may in general be negative. Take for example the Fii type quiver

$$
S=\left(\begin{array}{rrrr}
1 & -2 & 5 & -3 \\
0 & 1 & -7 & 5 \\
0 & 0 & 1 & -2 \\
0 & 0 & 0 & 1
\end{array}\right)
$$

with gauge group ranks all $S U(N)$. This "gauge theory" has has $R\left(X_{13}\right)=R\left(X_{24}\right)=-1 / 4$ according to our formulae. Gauge invariant, antisymmetric products of these $X_{13}$ and $X_{24}$ correspond to dibaryonic operators and naively would have a negative R-charge. As these 
dibaryons are chiral primaries, they would also have a negative conformal dimension in violation of unitarity. ${ }^{2}$

\subsection{Mutation versus Seiberg Duality}

We investigate the effects of mutation and Seiberg Duality on a four node quiver. Up to now, we have considered $L^{D}$ and $R^{D}$ type mutations. To mutate a collection to obtain a different collection, we operate with $R$ and $L$ type mutations on the helix $\mathcal{H}$. In this way, we can be sure that the ranks of the gauge groups stay positive. If we were to mutate the dual gauge theory collection, where some of the sheaves have negative rank, it is not a priori clear how to choose the signs to satisfy chiral anomaly cancellation.

For the four node quivers, then, the geometric collection $\mathcal{E}^{G}=\left(E_{4}^{\vee}, E_{3}^{\vee}, E_{2}^{\vee}, E_{1}^{\vee}\right)$ generates the helix $\mathcal{H}$. Consider $L_{E_{4}^{\vee}} E_{3}^{\vee}$. Under such a mutation, the entries of $S$ become

$$
\begin{array}{r}
a \rightarrow a, \quad b \rightarrow c-b f, \quad c \rightarrow \pm b, \\
d \rightarrow e-d f, \quad e \rightarrow \pm d, \quad f \rightarrow \mp f .
\end{array}
$$

One may also consider $R_{E_{3}^{\vee}} E_{4}^{\vee}$ where

$$
\begin{gathered}
a \rightarrow a, \quad b \rightarrow \pm c, \quad c \rightarrow b-c f, \\
d \rightarrow \pm e, \quad e \rightarrow d-e f, \quad f \rightarrow \mp f .
\end{gathered}
$$

Applying these transformations to $\mathrm{Ai}, \mathrm{Fi}$, Aii, etc., one finds that mutations in general map $A$ type quivers to both $A$ and $F$ type quivers and similarly for $F$ type.

However, it is worthwhile to look more closely. In [12], it was pointed out that Seiberg duality sometimes corresponds to a mutation. Seiberg duality for us will mean a particular combinatoric action on the quiver. A careful treatment requires knowledge of the superpotential which we lack in general. Combinatorially, we specify a node on which to dualize. We change the rank of the gauge group at that node from $N_{c}$ to $N_{f}-N_{c}$. For example, for

\footnotetext{
${ }^{2} \mathrm{~K}$. Intriligator and R. Tatar have suggested in private communication that these negative R-charges might indicate the appearance of an accidental $\mathrm{U}(1)$ symmetry in the gauge theory. In simpler gauge theories where this problem occurs, for example SQCD, as a result of the symmetry the troublesome gauge invariant operators decouple from the interacting theory and become free chiral superfields. (See [30] for a recent discussion of this phenomenon.) However, it is not clear to the author what would become of these troublesome dibaryons.
} 
an $A i$ quiver, dualizing on node 4 would send $N r_{4} \rightarrow N\left(c r_{1}-r_{4}\right)$. For every bifundamental that transformed under $S U\left(N_{c}\right)$, we introduce a new bifundamental with the opposite chirality that transforms under $S U\left(N_{f}-N_{c}\right)$. For $A i$ and node 4, this introduction would send $c \rightarrow-c, e \rightarrow-e$, and $f \rightarrow-f$. Finally, we combine the old bifundamentals that transformed under $S U\left(N_{c}\right)$ into mesonic type operators. These mesonic operators look like new bifundamentals and ensure chiral anomaly cancellation. The superpotential is critical at this step; unless the superpotential allows these bifundamentals to be integrated out properly, bidirectional arrows may exist in the Seiberg dual theory, spoiling a description using exceptional collections. Assuming the appropriate superpotential, for $A i$ and node 4, these mesonic operators send $b \rightarrow b-c f$ and $a \rightarrow a-c e$.

Now Seiberg duality does not respect the ordering of the quiver. After a Seiberg duality, the quiver must usually be reordered in order to correspond to an exceptional collection.

Returning to our investigation of the relation between Seiberg duality and mutation, recall that in general each node in a four node quiver is connected to the other three nodes by three arrows; not all the arrows point in the same direction.

Assume only one arrow points into the node. If this arrow comes from a node that is a nearest neighbor to the right in the gauge theory collection $\mathcal{E}^{Q}$, then a left mutation over the corresponding node in the geometric collection will correspond to a Seiberg duality.

Assume only one arrow points away from the node. If this arrow goes to a node that is a nearest neighbor to the left in the gauge theory collection $\mathcal{E}^{Q}$, then a right mutation over this neighboring node in the geometric collection corresponds to Seiberg duality.

Moreover, the mutation whether left or right will always be a division in these special circumstances.

In general, there are eight possible mutations, left and right for each of the four nodes. However, there are only four ordinary Seiberg dualities, one for each node. Looking closely at the $A$ and $F$ type quivers we see that for $A$ type, four of the eight mutations correspond to Seiberg dualities. In other words, Seiberg duality can always be thought of as a mutation for the $A$ type quiver. Specifically, for the $A$ type quiver, every node has a single in or out arrow pointing to or away from a neighboring node in the collection. Moreover, the single in arrow will come from the right and the single out arrow will always point to the left in $\mathcal{E}^{Q}$.

For the $F$ type quiver, only two of the eight mutations correspond to Seiberg dualities. 
The remaining two Seiberg dualities don't correspond to mutations. More precisely, assuming that no arrows become bidirectional after the duality, we cannot satisfy the constraint (333) for any ordering after the duality. It seems likely that if these $F$ type quivers have a sensible gauge theory interpretation, then these remaining two Seiberg dualities produce quivers with bidirectional arrows, i.e. quivers that cannot possibly come from exceptional collections.

One remaining intriguing fact here is that Seiberg duality, when it corresponds to a mutation, maps $A$ type quivers to $A$ type quivers and $F$ type to $F$ type.

Based on the negative R-charges and the strange behavior under Seiberg duality, it is very tempting to conclude that $F$ type quivers are not allowed. However, we lack a geometric understanding of why these $F$ type quivers should be ruled out. From the exceptional collection point of view, one geometric collection seems just as good as another. ${ }^{3}$

As mentioned in the introduction, it is tempting to generalize from our experience with four node quivers. We define a well split quiver to be such that for any node $i$, all the nodes in-going into $i$ can be placed to the right in the $\mathcal{E}^{Q}$ and all the outgoing nodes with respect to $i$ to the left in $\mathcal{E}^{Q}$. For such a quiver, Seiberg duality should always correspond to a left mutation of $E_{i}^{G}$ over all the in-going nodes in $\mathcal{E}^{G}$ [13, 16]. Furthermore, one hopes that the Seiberg dual of a well split quiver is again well split. In this language, $A$ type four-node quivers are well split. In constrast, a quiver which does not satisfy this property we call ill split because the in-going and outgoing nodes do not split in a way that respects the ordering of the exceptional collection. $F$ type quivers are ill split quivers.

\section{Toward a General Understanding}

In proving cyclic invariance of the quiver ordering, deriving the formula for the ranks of the gauge groups in terms of paths in the quiver, classifying four node quivers, and checking the value of $\operatorname{Tr} R^{3}$ for four node quivers, we have taken a few steps toward understanding the connections between exceptional collections, del Pezzo gauge theories, and AdS/CFT correspondence. However, there remains a long list of things to do. In hopes of inspiring the reader, we briefly describe a few of these items.

Number one on this list is obtaining a better understanding of $F$ type quivers, or more

\footnotetext{
${ }^{3}$ To the author's knowledge, the first example of such an $F$ type quiver appeared in [31].
} 
generally, ill split quivers. For four node quivers, we saw that the set of $A$ type quivers was closed under Seiberg duality. Thus, we can consider only $A$ type quivers if we are interested in their behavior under RG flow. More generally, it would be interesting to prove that well split quivers are closed under Seiberg duality. Also interesting would be some kind of geometric demonstration that $F$ type and ill split quivers are not allowed as gauge theories.

Number two is an analysis of the behavior of these gauge theories under RG flow. How generic is the KS flow of [19]? Can we obtain an analytic understanding of the duality walls in [32]? We plan to return to these questions in 20].

Number three would be to understand how exceptional collections relate to another way of thinking of Seiberg duality considered in [33, 34, 35]. In these papers, Seiberg duality is related to tilting equivalences of certain derived categories.

Finally, there are a large number of technical details which need to be resolved. Here are three such details. One ought to be able to prove that the R-charge formula (9) corresponds to the maximization of $a_{c}$ principle derived by [36. Second, one ought to be able to use the R-charges to check that $\operatorname{Tr} R^{3}=24 / K^{2}$ for a general del Pezzo quiver. Third, one should show that the rank two condition and the trace condition $\operatorname{Tr} S S^{-T}=n$ on $S$ are not only necessary but sufficient for $S$ to correspond to an exceptional collection.

Hopefully, some of these issues will be resolved soon.

\section{Acknowledgments}

It is a pleasure to thank S. Franco, A. Hanany, Y.-H. He, K. Intriligator, J. McKernan, M. Rangamani, M. Spradlin, and R. Tatar for discussion. The author would like to thank J. Walcher for comments on the manuscript. The author would also like to thank Berkeley, where part of this work was prepared, for hospitality. This research was supported in part by the National Science Foundation under Grant No. PHY99-07949.

\section{References}

[1] D. Morrison and R. Plesser, "Non-Spherical Horizons, I," Adv. Theor. Math. Phys. 3 (1999) 1, hep-th/9810201. 
[2] B. S. Acharya, J. M. Figueroa-O'Farrill, C. M. Hull, and B. Spence, "Branes at conical singularities and holography," Adv. Theor. Math. Phys. 2 (1999) 1249, hep-th/9808014.

[3] I. R. Klebanov and E. Witten, "Superconformal field theory on three-branes at a CalabiYau singularity," Nucl. Phys. B536 (1998) 199, hep-th/9807080.

[4] A. Kehagias, "New Type IIB Vacua and Their F-Theory Interpretation," Phys. Lett. B435 (1998) 337, hep-th/9805131

[5] J. Maldacena, "The Large N limit of superconformal field theories and supergravity," Adv. Theor. Math. Phys. 2 (1998) 231, hep-th/9711200.

[6] S.S. Gubser, I.R. Klebanov, and A.M. Polyakov, "Gauge theory correlators from noncritical string theory," Phys. Lett. B428 (1998) 105, hep-th/9802109.

[7] E. Witten, "Anti-de Sitter space and holography," Adv. Theor. Math. Phys. 2 (1998) 253, hep-th/9802150.

[8] C. Beasley, B. R. Greene, C. I. Lazaroiu and M. R. Plesser, "D3-branes on partial resolutions of abelian quotient singularities of Calabi-Yau threefolds," Nucl. Phys. B566 (2000) 599, hep-th/9907186.

[9] C. E. Beasley and M. Ronen Plesser, "Toric Duality is Seiberg Duality," JHEP 0112 (2001) 001, hep-th/0109053

[10] B. Feng, A. Hanany and Y. H. He, "D-brane gauge theories from toric singularities and toric duality," Nucl. Phys. B595 (2001) 165, hep-th/0003085.

[11] B. Feng, A. Hanany and Y. H. He, "Phase structure of D-brane gauge theories and toric duality," JHEP 0108 (2001) 040, hep-th/0104259

[12] F. Cachazo, B. Fiol, K. A. Intriligator, S. Katz and C. Vafa, "A geometric unification of dualities," Nucl. Phys. B 628 (2002) 3, hep-th/0110028

[13] M. Wijnholt, "Large volume perspective on branes at singularities," hep-th/0212021.

[14] B. Feng, S. Franco, A. Hanany, and Y. H. He, "Unhiggsing the del Pezzo," JHEP 0308 (2003) 058, hep-th/0209228. 
[15] A. Iqbal and A. Hanany, "Quiver Theories from D6-branes via Mirror Symmetry," JHEP 0204 (2002) 009, hep-th/0108137

[16] C. P. Herzog and J. Walcher, "Dibaryons from Exceptional Collections," JHEP 0309 (2003) 060, hep-th/0306298.

[17] C. P. Herzog and J. McKernan, "Dibaryon Spectroscopy," JHEP 0308 (2003) 054, hep-th/0305048.

[18] K. Intriligator and B. Wecht, "Baryon charges in 4D superconformal field theories and their AdS duals," hep-th/0305046.

[19] I. R. Klebanov and M. J. Strassler, "Supergravity and a confining gauge theory: Duality cascades and chiSB-resolution of naked singularities," JHEP 0008, 052 (2000) 052, hep-th/0007191.

[20] S. Franco, Y.-H. He, C. P. Herzog, J. Walcher, "Chaotic Duality in String Theory," hep-th/0402120.

[21] "Helices and vector bundles," Seminaire Rudakov. London Mathematical Society Lecture Note Series, 148. Cambridge University Press, Cambridge, 1990.

[22] B. V. Karpov and D. Yu. Nogin, "Three-block Exceptional Collections over del Pezzo Surfaces," Izv. Ross. Akad. Nauk Ser. Mat. 62 (1998), no. 3, 3-38; translation in Izv. Math. 62 (1998), no. 3, 429-463

[23] K. Hori, A. Iqbal and C. Vafa, "D-branes and mirror symmetry," hep-th/0005247.

[24] S. A. Kuleshov and D. O. Orlov, "Exceptional sheaves over del Pezzo surfaces," Izv. Ross. Akad. Nauk Ser. Mat. 58 (1994), no. 3, 53-87; translation in Russian Acad. Sci. Izv. Math. 44 (1995), no. 3, 479-513

[25] D. Anselmi, D. Z. Freedman, M. T. Grisaru and A. A. Johansen, "Nonperturbative formulas for central functions of supersymmetric gauge theories," Nucl. Phys. B526 (1998) 543, hep-th/9708042.

[26] D. Anselmi, J. Erlich, D. Z. Freedman and A. A. Johansen, "Positivity constraints on anomalies in supersymmetric gauge theories," Phys. Rev. D 57 (1998) 7570, hep-th/9711035. 
[27] M. Henningson and K. Skenderis, "The holographic Weyl anomaly," JHEP 9807 (1998) 023, hep-th/9806087.

[28] S. S. Gubser, "Einstein Manifolds and Conformal Field Theories," Phys. Rev. D 59 (1999) 025006, hep-th/9807164.

[29] A. Bergman and C. P. Herzog, "The volume of some non-spherical horizons and the AdS/CFT correspondence," JHEP 0201 (2002) 030, hep-th/0108020.

[30] D. Kutasov, A. Parnachev, and D. A. Sahakyan, "Central Charges and $U(1)_{R}$ Symmetries in $\mathcal{N}=1$ Super Yang-Mills," hep-th/0308071.

[31] B. Feng, A. Hanany, Y. H. He and A. Iqbal, "Quiver theories, soliton spectra and Picard-Lefschetz transformations," JHEP 0302 (2003) 056, hep-th/0206152.

[32] S. Franco, A. Hanany, Y.-H. He, and P. Kazakopoulos, "Duality Walls, Duality Trees and Fractional Branes," hep-th/0306092.

[33] D. Berenstein and M. R. Douglas, "Seiberg duality for quiver gauge theories," hep-th/0207027.

[34] V. Braun, "On Berenstein-Douglas-Seiberg duality," JHEP 0301 (2003) 082, hep-th/0211173.

[35] S. Mukhopadhyay and K. Ray, "Seiberg duality as derived equivalence for some quiver gauge theories," hep-th/0309191.

[36] K. Intriligator and B. Wecht, "The exact superconformal R-symmetry maximizes a," Nucl. Phys. B667 (2003) 183, hep-th/0304128. 